\title{
IMPLANTAÇÃO DA INSPEÇÃO VEICULAR REMOTA BASEADA EM DADOS DE OBD, UMA ALTERNATIVA AO CONTROLAR?
}

\author{
Oscar Javier Duque ${ }^{1}$, Guido Roewer Jr. ${ }^{2}$, Paolo Furlan ${ }^{2}$, Alberto Bucci ${ }^{2}$ \\ ${ }^{1}$ FEV Brasil Tecnologia de Motores \\ ${ }^{2}$ Magneti Marelli Sistemas Automotivos
}

duque@fev.com, guido.roewer@magnetimarelli.com, paolo.furlan@magnetimarelli.com, alberto.bucci@magnetimarelli.com

\section{RESUMO}

O presente trabalho visa mostrar a possibilidade de se implantar o monitoramento remoto dos dados de OBD tais como: coeficiente de desempenho dos monitores (In Use Monitor Performance Ratios, IUMPR), status da LIM (Lâmpada Indicadora de Mau Funcionamento) e dos códigos de falha armazenados na memória de avarias. Tais dados seriam enviados diretamente aos órgãos governamentais interessados e utilizados como uma inspeção veicular remota, uma alternativa às inspeções veiculares periódicas, e que possibilitaria a abrangência de todo o território nacional.

A implantação dos IUMPR apresenta um desafio na calibração OBD, uma vez que as regiões de funcionamento do motor nas quais as diagnoses OBD são ativadas deveriam ser ampliadas, aumentado assim o risco de falsas detecções. Por outro lado, caso as faixas de habilitação sejam muito estreitas, os coeficientes de performance seriam menores que o exigido pela normativa, podendo em casos extremos ocasionar um recall do veículo.

\section{INTRODUÇÃO}

Em 2014 foram apresentadas diversas atualizações nas legislações de emissões dos principais mercados do mundo (Figura 1), tais como a introdução da normativa EU6 juntamente com sua atualização OBD na Europa, a primeira fase do LEVIII e OBDIII nos Estados Unidos, e o início da legislação baseada nos limites de emissões definidos pelo Proconve L6 no Brasil. 


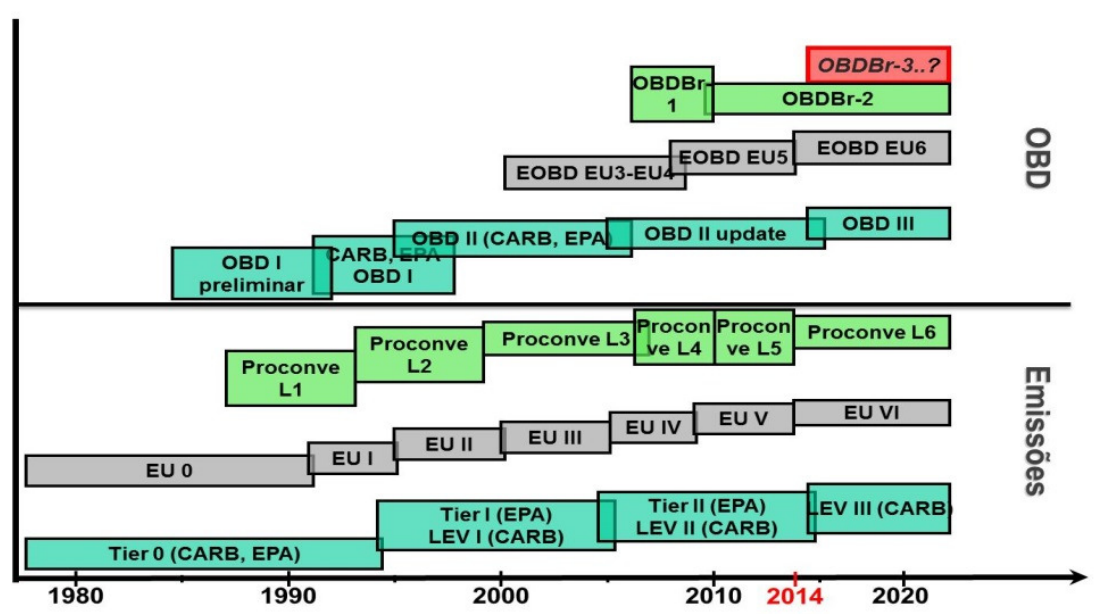

Figura 1. Evolução dos programas de emissões veiculares e diagnoses OBD [1]

A legislação OBD nos EUA continua ainda sendo referência para outros mercados em termos de complexidade e abrangência. As diferenças mais relevantes dos atuais sistemas OBD nos EUA e na Europa são apresentadas na Figura 2 na qual são comparadas com a atual legislação OBD brasileira. Pode-se verificar que o mercado brasileiro é o único no qual a normativa OBD foi implantada para percentuais de etanol de até $100 \%$. Um dos principais desafios para o setor automotivo brasileiro foi a implantação destes sistemas em um mercado no qual cerca de 90\% dos veículos leves vendidos são Flex. Isto implica em assegurar o correto funcionamento do sistema OBDBr-2 tanto pra gasolina como para etanol, mesmo que estes sejam combustíveis com características bem diferentes [1].

\begin{tabular}{|c|c|c|c|}
\hline & OBD-II (USA) & EOBD EU6 & $\mathrm{OBDBr}-2$ \\
\hline Diagnose Catalisador (NMHC) & $\sqrt{ }$ & $\sqrt{ }$ & $\sqrt{ }$ \\
\hline Diagnose Catalisador (NOx) & $\checkmark$ & $\sqrt{ }$ & $\mathrm{X}$ \\
\hline Diagnose resposta sonda Upstream & $\sqrt{ }$ & $\checkmark$ & $\sqrt{ }$ \\
\hline Diagnose resposta sonda Downstream & $\sqrt{ }$ & $\sqrt{ }$ & $\mathrm{x}$ \\
\hline Diagnose Misfire & $\checkmark$ & $\checkmark$ & $\sqrt{ }$ \\
\hline Diagnose Fuel System & $\checkmark$ & $\checkmark$ & $\mathrm{X}$ \\
\hline Diagnoses elétricas & $\checkmark$ & $\checkmark$ & $\checkmark$ \\
\hline Diagnose Ar secundário & $\checkmark$ & $\checkmark$ & $\mathrm{X}$ \\
\hline Diagnose Cylinder Imbalance & $\sqrt{ }$ & $\mathrm{x}$ & $\mathrm{X}$ \\
\hline In Use Monitor Performance Ratios (IUMPR) & $\sqrt{ }$ & $\checkmark$ & $\mathrm{X}$ \\
\hline Sistemas Flex (Álcool e Gasolina) & $\mathrm{X}$ & $\mathrm{X}$ & $\checkmark$ \\
\hline
\end{tabular}

Figura 2. Comparação dos programas OBD atuais nos EUA, Europa e Brasil.

Ao conferir as exigências do sistema OBD, é preciso levar em consideração as particularidades do etanol como combustível. Entre estas particularidades está o aumento na evaporação proveniente do cárter do motor ("blow by"), diferença de ponto de ignição e de frequência de comutação da sonda lambda em relação ao aplicado para o funcionamento do motor à gasolina. Entretanto, tecnologias já desenvolvidas no país permitem contornar possíveis problemas advindos de tais diferenças e tornar possível caminhar em direção a uma 
futura aproximação das normas relativas às diagnoses de bordo presentes nos mercados mais exigentes do mundo.

Nos EUA, diversas implantações têm sido feitas com a finalidade de maximizar os benefícios dos sistemas OBD, e minimizar os custos de inspeção para os proprietários dos veículos. Sendo assim, muitos estados têm substituído os programas de inspeção e manutenção (I/M) convencionais, pela inspeção do sistema OBD (falhas detectadas, status da LIM, etc.).

Uma das propostas mais debatidas atualmente é a implantação de uma inspeção remota do sistema OBD (OBDIII), usando alguma tecnologia de transmissão de dados. Este tipo de sistema baratearia os custos dos programas de inspeção. No Brasil, um sistema equivalente poderia ser utilizado para abranger toda a frota automotiva. A informação transmitida poderia incluir o status da LIM e falhas armazenadas, assim como também a frequência com que os monitores OBD têm sido concluídos (IUMPR). Este tipo de sistema exigiria uma precisão e robustez ainda maior do sistema OBD, uma vez que falsas detecções poderiam ser identificadas mais rapidamente, o que poderia levar a recalls dos veículos.

\section{A AVAliação do CONTROLAR COMO MÉtodo de REDUÇÃo dAS EMISSÕES}

O sistema Controlar de inspeções periódicas vinha conseguindo resultados positivos, com evidente impacto favorável no meio ambiente. Os resultados do Controlar em São Paulo no ano 2011 são apresentados na Figura [2].

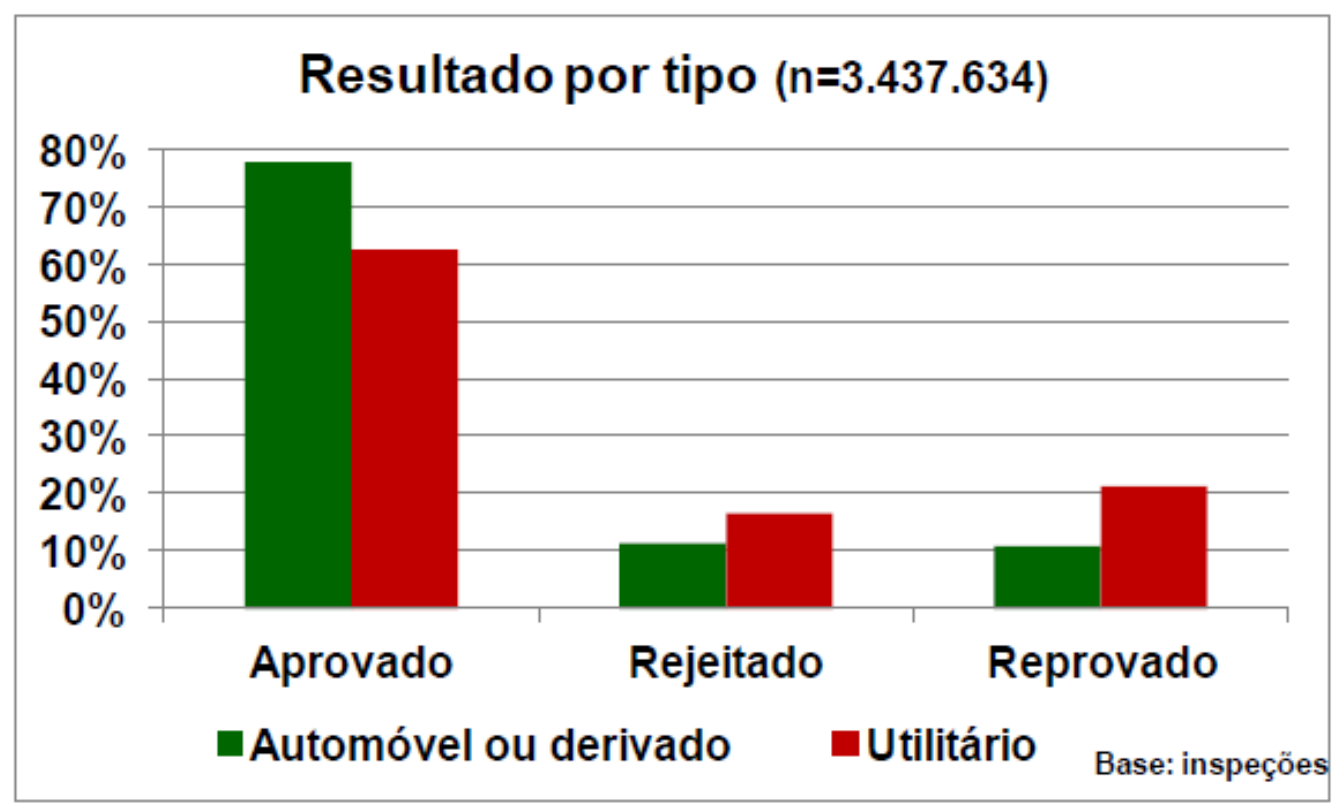

Figura 3. Resultado das inspeções Controlar em 2011 [2]

Todavia o caminho para um melhor controle das emissões dos veiculos e o respeito das normativas em geral é ainda longo quando se leva em consideração o modelo dos mercados americano e europeu. Para isso foi concebida a resolução CONAMA 418/2009, na qual se 
define que o IBAMA deve coordenar os estudos para identificar de forma mais eficiente a Inspeção e Manutenção (I/M) com procedimentos alinhados com as novas tecnologias para veículos incluindo a possibilidade de integrar os sistemas de controle OBD.

Para melhor entender essa possibilidade de integração no Brasil, foi ja realizado um estudo [3] que demonstra a correlação entre as falhas e limites OBDBr-2, e os limites na I/M. Em outras palavras, é razoável esperar que um veículo com a luz indicadora de mau funcionamento (LIM) acesa vai ultrapassar os limites de aceitação do teste I/M e vice-versa.

De acordo com estudo mencionado, a normativa OBDBr-2 parece ser mais rigorosa que a I/M em relação aos limites de emissões. De qualquer modo, a recente introdução na Instrução Regulamentar 6/2010 da inspeção visual no teste I/M da LIM está na direção de uma maior proteção do ambiente.

Sendo que o principal objetivo do sistema OBD é determinar se o sistema de controle de emissões está funcionando corretamente, muitos países já estão usando a verificação OBD como um substituto das emissões do escape como, por exemplo, a Diretiva comunitária 96 196/CE do conselho da União Europeia e a EPA 40 CFR Parte 51 e 85 para os Estados Unidos [3].

A hipótese de introduzir a norma $\mathrm{OBDBr}-2$ na avaliação I/M deve levar também em consideração a dificuldade na execução do teste completo OBD, no uso normal de um veículo. Para tanto há o coeficiente IUMPR, que é um índice da capacidade do sistema de executar e completar os monitores OBD. Deste modo se faz necessário compreender melhor o comportamento dos condutores em geral. A incapacidade de alcançar o valor mínimo de IUMPR poderia resultar em um recall do produto nas normativas atuais americanas e europeias. Uma ferramenta que quantifica a possibilidade de completar a execução das diagnoses em função do tipo de rodagem, da estrutura viária e do estilo de condução do motorista foi apresentada em [4]. O objetivo é a criação de um sistema de diagnóstico e sua calibração de modo a se conseguir satisfazer todas as possíveis condições encontradas no mercado de venda do veículo.

Uma possível desvantagem do sistema de inspeção remota OBD em lugar da I/M convencional como a feita pelo Controlar, seria a perda da oportunidade de uma inspeção visual do veículo, que poderia detectar vazamentos que também são formas de emissões, bem como adulterações no sistema $\mathrm{OBD}$ ou de quaisquer outros componentes do veículo não permitidas pela lei.

\section{COEFICIENTES DE PERFORMANCE DOS MONITORES OBD (IUMPR)}

Embora alguns dos componentes do veículo pudessem ser monitorados continuamente pelo sistema OBD, outros necessitam condições específicas de habilitação, de modo que os resultados das diagnoses sejam confiáveis. Estas condições devem ser definidas de modo a atender aos seguintes requisitos: 
- Garantir a detecção confiável das falhas, evitando a não detecção de falhas reais e falsas indicações de falha.

- Assegurar que o monitoramento vai ocorrer em condições que possam ser razoavelmente reproduzidas em condições normais de funcionamento do veículo.

- Assegurar que o monitoramento ocorrerá durante o ciclo NBR6601 para efeitos de homologação do sistema.

Para quantificar esta frequência de monitoramento, a CARB introduziu em 2005 na normativa OBDII o conceito de "In Use Monitor Performance Ratio (IUMPR ou IUPR)", sendo também introduzido em 2012 na normativa EU5+. O IUMPR pode ser definido como a indicação de quantas vezes um monitor específico está habilitado durante o funcionamento do veículo [5]:

$$
I U M P R=\frac{\text { Numerador }}{\text { Denominador }}
$$

Numerador: Número de ciclos de condução em que uma falha poderia ter sido detectada

Denominador: Número de ciclos de condução, que atendem às seguintes condições regulamentadas:

- Tempo acumulado com motor ligado $>600 \mathrm{~s}$

- Velocidade acumulada do veículo $>40 \mathrm{~km} / \mathrm{h}$ por mais de $300 \mathrm{~s}$

- Tempo em marcha lenta $>30 \mathrm{~s}$

- Temperatura ambiente $>-7^{\circ} \mathrm{C}$

- Altitude $<2500 \mathrm{~m}$

O denominador é incrementado em 1 sempre que o ciclo de condução atende a estas condições.

A implementação dos IUMPR apresenta um desafio na calibração OBD, uma vez que as regiões de habilitação das diagnoses deveriam ser ampliadas, aumentado assim o risco de falsas detecções (Figura 4). Por outro lado, caso as faixas de habilitação sejam muito estreitas, os coeficientes de performance seriam menores que o exigido pela norma, podendo em casos extremos ocasionar um recall do veículo.

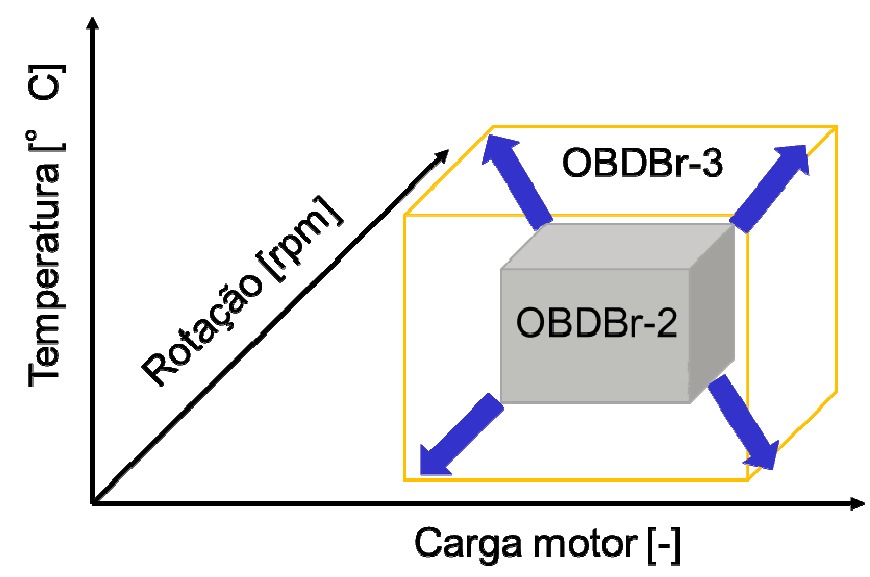

Figura 4. Ampliação das zonas de diagnoses OBD 
Os coeficientes atuais mínimos definidos na normativa OBDII e EU6 são 0,336 para as diagnoses de catalisador e dos sensores de oxigênio. Para a implantação destes coeficientes na normativa brasileira, as condições para incrementar o denominador precisariam ser reformuladas, considerando-se os critérios de desativação temporária do sistema OBD próprios dos sistemas flex e permitidos pela legislação, tais como:

- Durante a fase de reconhecimento e aprendizado do tipo de combustível;

- Durante a fase de evaporação do combustível contaminante do óleo lubrificante (Blow-by).

Diferentes pesquisas têm se realizado nos EUA e Europa para tentar definir os estilos de condução e sua semelhança com os ciclos de condução estandardizados para ensaios de homologação veicular. Por exemplo, uma frota avaliada na Europa mostrou predominantemente velocidades altas com acelerações suaves, assim como períodos curtos, porém frequentes, em marcha lenta. Por outro lado, a frota avaliada nos EUA produziu longos períodos em marcha lenta, mas pouco frequentes, assim como acelerações mais bruscas, porém uma vez alcançada a velocidade desejada, esta permaneceu constante por tempos maiores que na Europa [4]. Estas pesquisas ajudam os órgãos governamentais a definir os valores mínimos dos coeficientes de desempenho dos monitores OBD.

Outra comparação feita é a diferença entre os ciclos de emissões padrão nos EUA e na Europa. É notório que os trechos de velocidade constante e marcha lenta são maiores no ciclo europeu, sendo o ciclo americano bem mais dinâmico apresentando mais acelerações. Isto permite deduzir que, em realidade, os ciclos de condução não refletem a realidade da forma de condução em seus territórios.

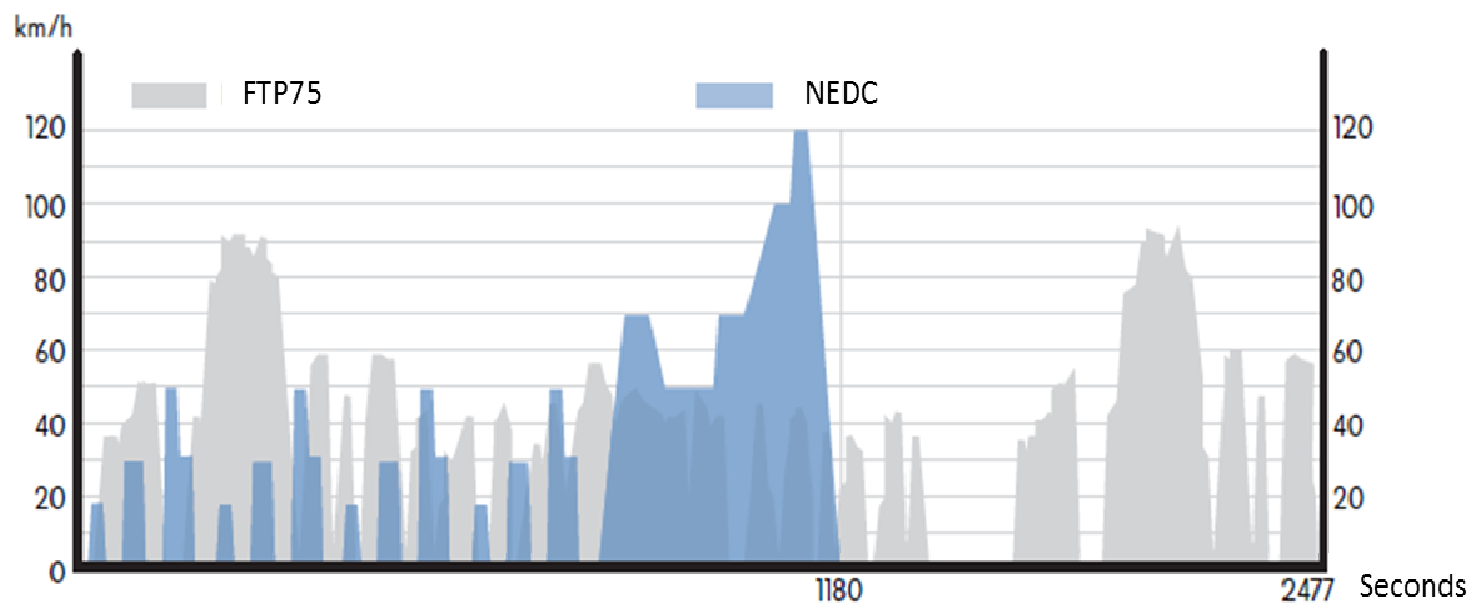

Figura 5. Comparação dos ciclos FTP75 e NEDC [6]

Não existe, por enquanto, um estudo similar no Brasil. Porém, pode-se inferir, de acordo com a estrutura da malha rodoviária, os limites de velocidade, e a potência média dos veículos no mercado nacional, que a condução no Brasil se assemelha mais àquela europeia, ainda que o ciclo padrão para homologação utilizado seja similar ao ciclo americano, FTP75. Do ponto de vista OBD há uma complexidade maior em razão do ciclo padrão de homologação, por outro lado, a calibração dos monitores no Brasil estaria naturalmente mais próxima de atender aos requisitos relativos à forma de condução semelhante à europeia. 


\section{RESULTADOS DA PESQUISA DOS IUMPR EM FROTA}

Com o objetivo de compreender o cenário local atual dos veículos flex equipados com sistema OBD com relação aos coeficientes de performance, foram levantados dados em campo sobre o comportamento dos monitores do sensor de oxigênio e da eficiência do catalisador, com veículos e calibrações homologadas e em produção. Para a pesquisa foram usados o mesmo modelo de veículo e calibração, em dois tipos padronizados de percurso:

- O primeiro percurso continha trechos de rodovia, estradas vicinais e trechos urbanos.

- O segundo percurso continha trechos apenas urbanos e estradas vicinais.

A Figura 6 apresenta os trechos eleitos para a pesquisa:

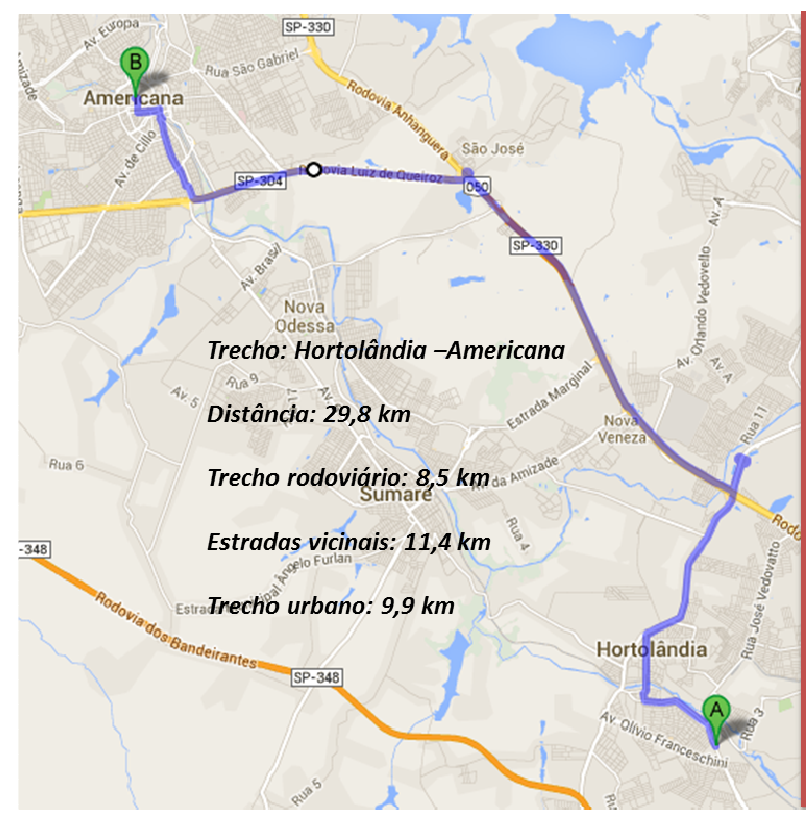

(a)

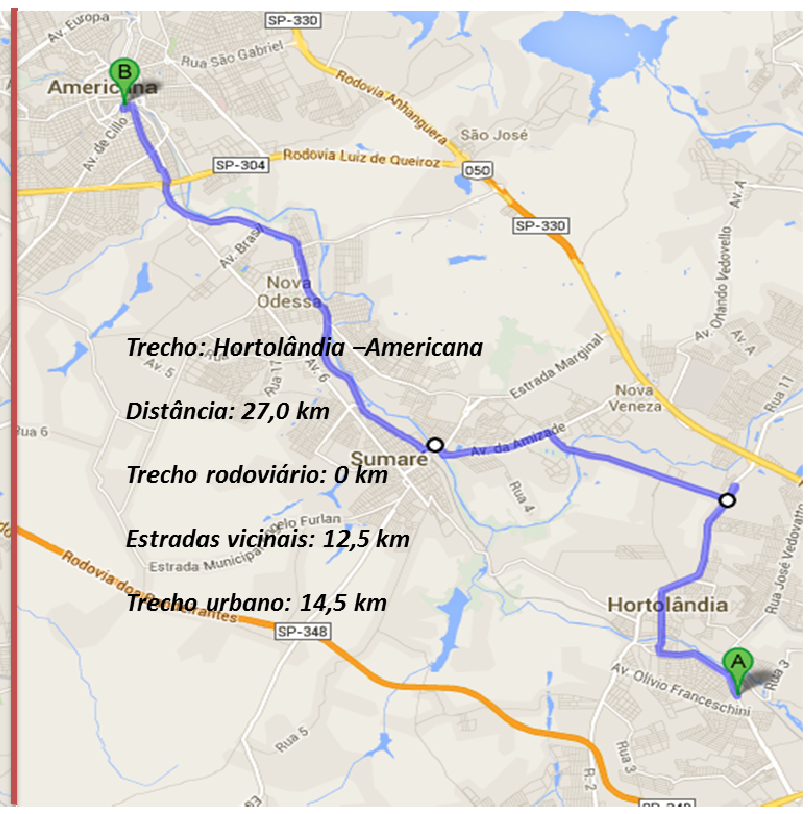

(b)

Figura 6. Ciclo Hortolândia-Americana para estudo do IUMPR; (a) Trecho rodoviário, vicinal e urbano; (b) Trecho somente vicinal e urbano [7]

Embora estes trechos não sejam muito representativos se comparados aos ciclos de condução em cidades de grandes fluxos veiculares como São Paulo, a qual concentra a maior frota veicular brasileira, foram utilizados por ter certa semelhança com as condições encontradas no ciclo NBR6601.

Um total de quatro diferentes veículos do mesmo modelo foram utilizados nesta pesquisa, todos utilizando uma calibração padrão com os algoritmos para o cálculo dos IUMPR habilitados. De modo geral são visualizadas na Figura 7 as regiões nominais de ativação das diagnoses de sonda e catalisador. A Figura 8 apresenta a distribuição de velocidade e rotação do motor nos dois trechos escolhidos. 


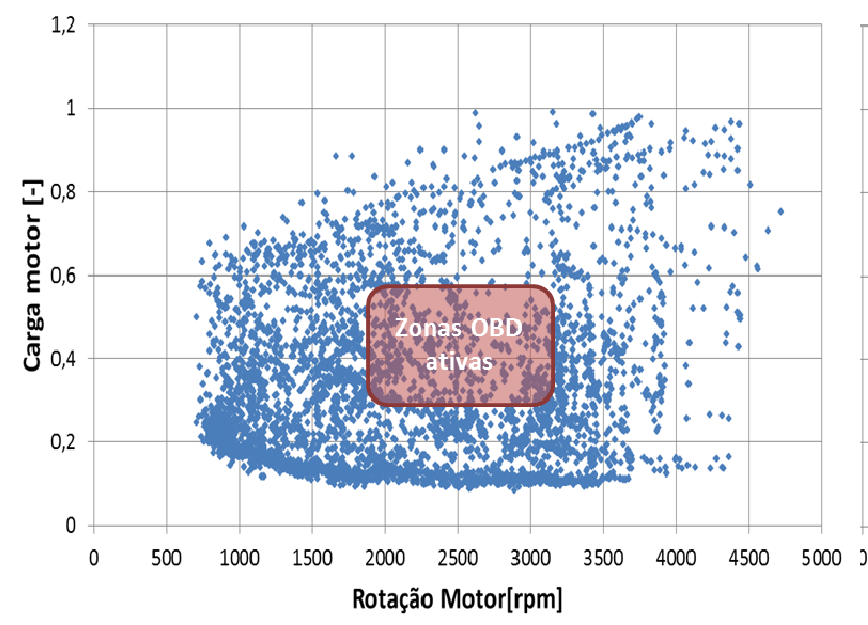

a.

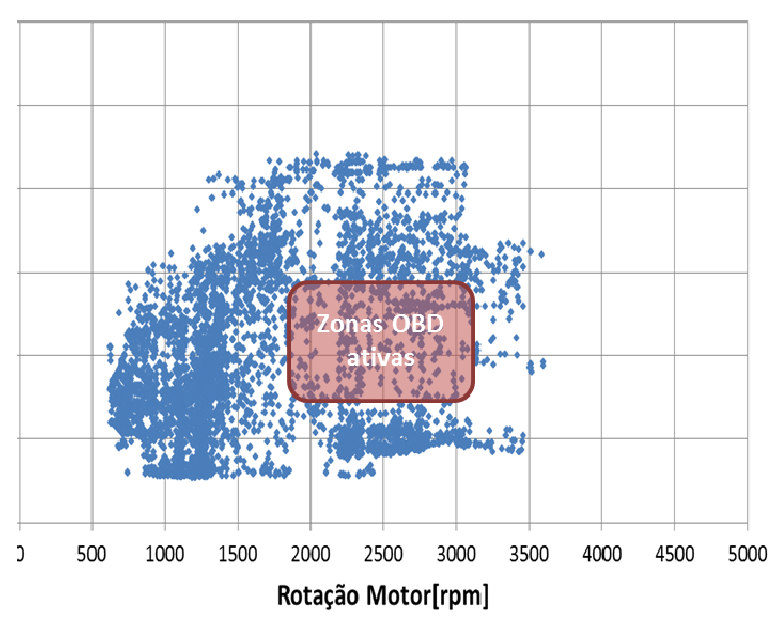

b.

Figura 7. Regiões de rotação e carga no trecho Hortolândia-Americana com Rodovia (a.) e sem Rodovia (b.)
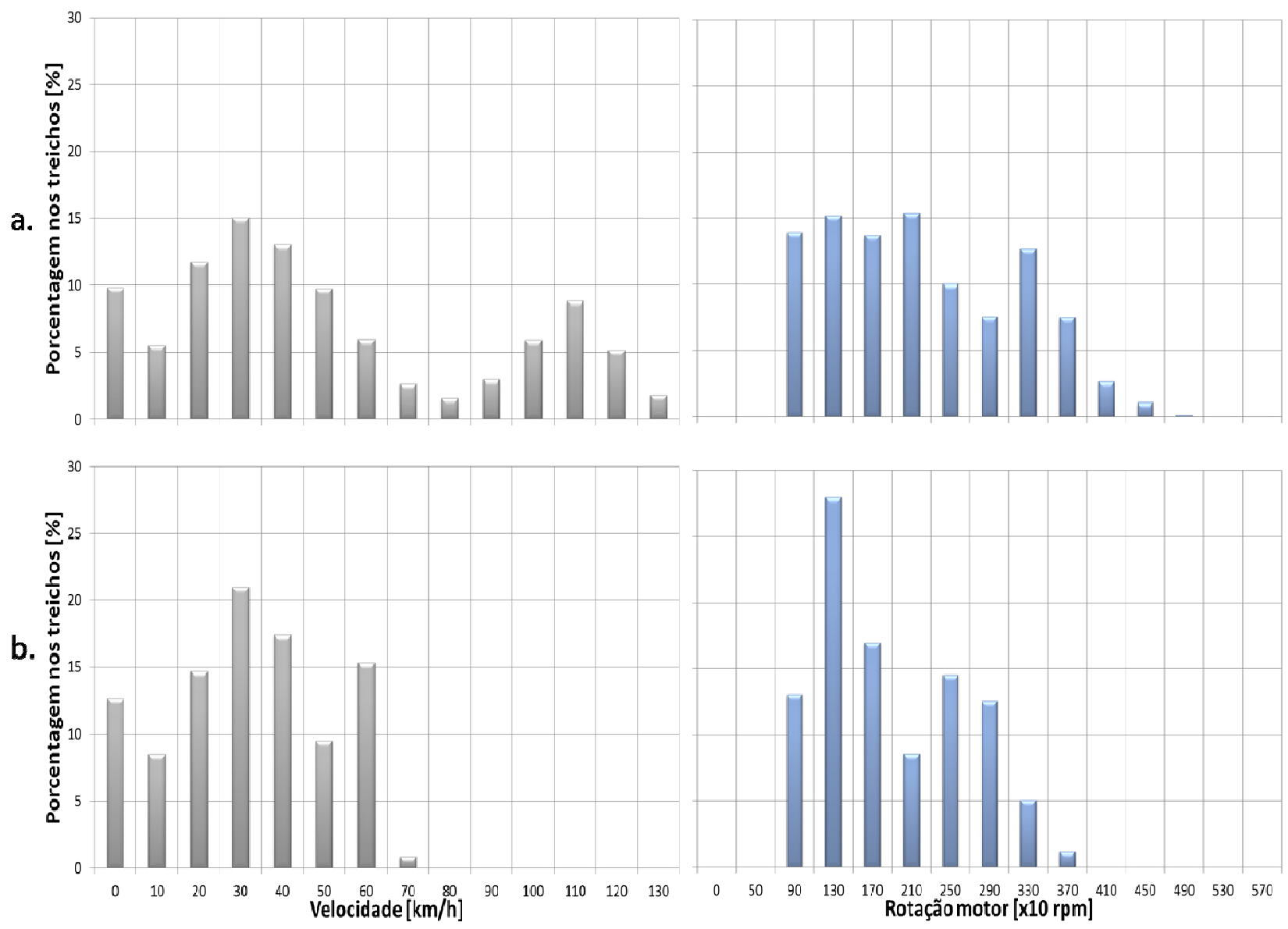

Figura 8. Distribuição da velocidade do veículo e rotação do motor durante o trecho Hortolândia-Americana com Rodovia (a.) e sem Rodovia (b.)

Nota-se nas figuras 7 e 8 que durante a maior parte dos trechos, os monitores OBD de sonda e catalisador estão desativados em razão da rotação do motor mais frequente ser na região de 
1200rpm para o trecho sem rodovia, e entre 1200 e 2100rpm para o trecho com rodovia. A zona de velocidade entre 80 e $90 \mathrm{~km} / \mathrm{h}$ apresenta baixa frequência de ocorrência no trecho com rodovia e nenhuma ocorrência no trecho sem rodovia, sendo esta zona uma das mais utilizadas para habilitação das diagnoses no ciclo NBR6601.

Todos os resultados dos IUMPR para os quatro diferentes veículos, nos dois trechos, estão resumidos nas figuras 9 e 10, apresentando a relação entre os trips válidos (Denominador geral), e o número de vezes em que as diagnoses de sonda e catalisador concluíram, ou seja, poderiam ter detectado uma falha OBD.

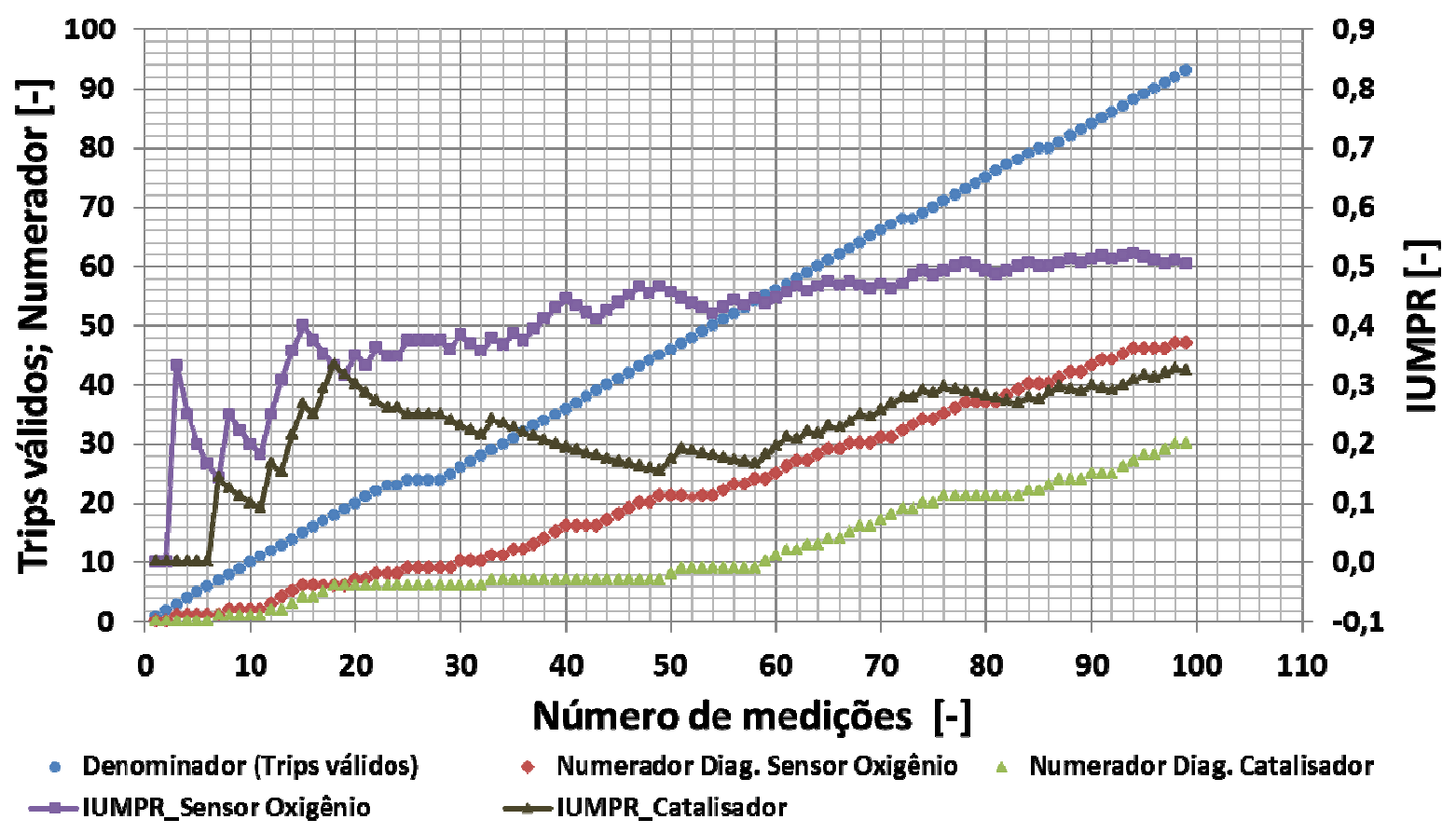

Figura 9. Resultados da frequência de conclusão das diagnoses, Trecho com Rodovia.

Da Figura 9 podem ser inferidos os valores dos coeficientes de performance dos monitores analisados no trecho com rodovia:

$$
\begin{aligned}
& I U M P R_{-} \text {Sensor_Oxigênio }=\frac{\text { Num_Sensor }}{\text { Denominador }}=0,505 \\
& I U M P R_{-} \text {Catalisador }=\frac{\text { Num_Catalisador }}{\text { Denominador }}=0,323
\end{aligned}
$$




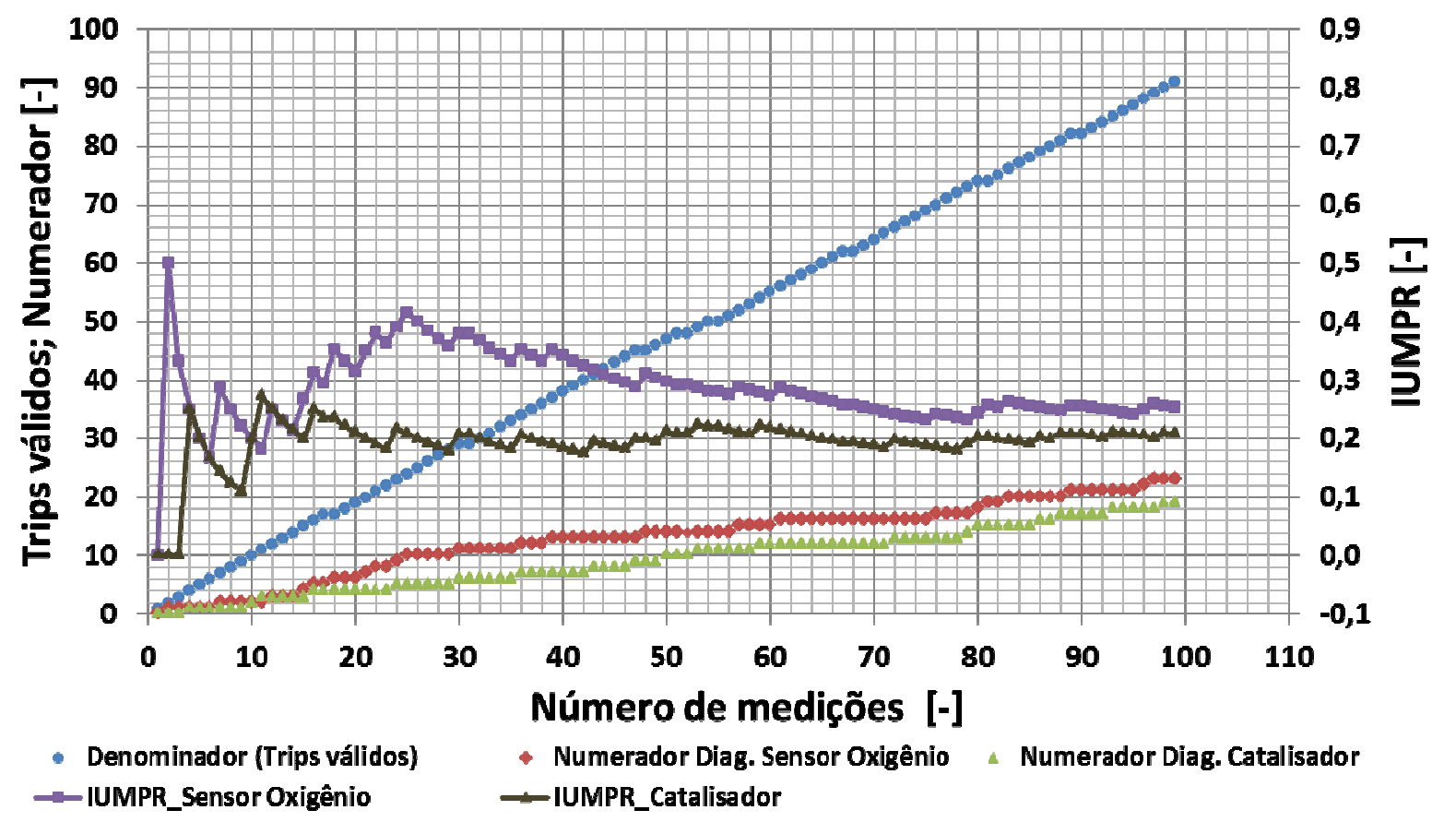

Figura 10. Resultados da frequência de conclusão das diagnoses, Trecho sem Rodovia.

De igual modo os valores dos coeficientes de performance para o trecho sem rodovia sao:

$$
\begin{aligned}
& I U M P R_{-} \text {Sensor_Oxigênio }=\frac{\text { Num_Sensor }}{\text { Denominador }}=0,253 \\
& I U M P R_{-} \text {Catalisador }=\frac{\text { Num_Catalisador }}{\text { Denominador }}=0,209
\end{aligned}
$$

Ou seja, o IUMPR para o monitor do sensor de oxigênio teve uma diminuição de aproximadamente $50 \%$ entre o trecho com rodovia e sem rodovia. Para o monitor do catalisador, esta diminuição é de $45 \%$.

A Figura 11 apresenta os valores médios de tempo de ativação do monitor do sensor de oxigênio. Há que se notar que o maior número de ocorrências é 0s de ativação do monitor (monitor desativado), seguido 1 e $2 \mathrm{~s}$ de ativação do monitor em ambos os trechos. Entretanto, apenas valores de ativação maiores de $7 \mathrm{~s}$ podem ser considerados válidos, pois a probabilidade de completar um cálculo é bastante alta. Sendo assim, percebe-se a probabilidade maior de completar um cálculo do monitor OBD durante o trecho com rodovia. 


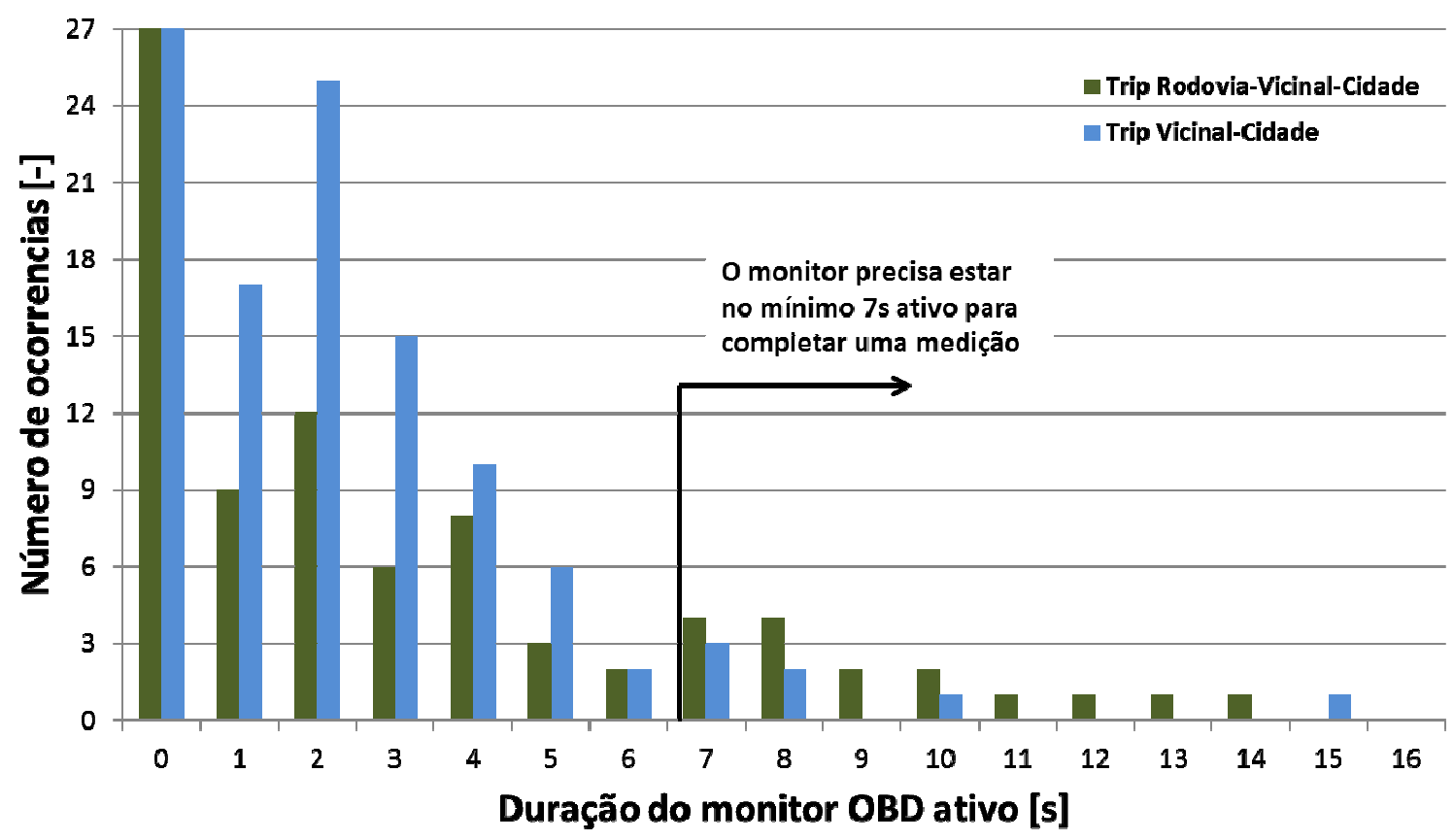

Figura 11. Frequência de habilitação do monitor do sensor de oxigênio e tempo de monitor ativo durante os dois trechos

Tomando como referência os valores mínimos dos IUMPR das mais exigentes normativas, a americana e a europeia, pode-se afirmar que os veículos estudados neste levantamento, poderiam cumprir uma legislação similar sem a necessidade de grandes alterações na calibração. Porém, este tipo de pesquisa também deveria ser realizado em trechos urbanos de maior concentração automotiva, como na grande São Paulo e será assunto de uma futura pesquisa.

\section{TRANSMISSÃO DOS DADOS OBD, POSSIBILIDADES E DESAFIOS.}

Nos Estados Unidos já se discute o que se vem chamando de OBD III. Esta evolução da norma OBD traria a implantação da inspeção veicular remota, baseada apenas em OBD. Esta seria uma alternativa de menor custo para os proprietários de veículos, pois não seria mais necessário o frequente deslocamento até os postos de fiscalização, representando uma economia de tempo e combustível, por si só uma redução nas emissões do veículo.

Existem no mercado diversas tecnologias disponíveis para a transmissão de dados, tais como: rádio frequência, redes de celular, wi-fi, Bluetooth, entre outras.

Atualmente, a tecnologia de maior abrangência é a rede de celulares. Entretanto, haveria um custo elevado, pois teriam que ser criadas bandas específicas para esta finalidade ou seriam utilizadas bandas compartilhadas com a telecomunicação, que em muitas regiões estão saturadas ou muito próximas disto, além de ser uma tecnologia que permite o tráfego de grandes volumes de informação, o que não é necessário para a finalidade aqui apresentada.

Uma alternativa de baixo custo seria a tecnologia Zigbee [8], que utiliza transmissores e receptores de curto alcance. Neste caso, os veículos sairiam de fábrica com transmissores, e 
ao passarem por "portais receptores", instalados em vias de grande fluxo de veículos e locais como praças de pedágio, enviariam informações sobre o veículo, tais como:

- VIN (Vehicle Identification Number)

- Status da LIM (Lâmpada indicadora de Mau Funcionamento)

- Códigos de falha armazenados

- Distância percorrida com a LIM acesa

- IUMPR

Para a finalidade a qual se destina, não é necessário que se envie a localização, data e o horário da transmissão, evitando que a privacidade do usuário seja violada. Porém, haveria a possibilidade de alguns veículos não transitarem por regiões cobertas pelos "portais receptores". Esta fragilidade seria praticamente eliminada com a instalação de receptores em veículos públicos, tais como: ônibus, ambulâncias, viaturas de polícia entre outros veículos a serviço do Estado. Deste modo, ao entrarem no alcance dos transmissores instalados nos veículos particulares, os veículos públicos receberiam as informações e as descarregariam ao passar pelos "portais receptores" ou quando regressassem para suas garagens.

As informações transmitidas ficariam armazenadas em uma central, cujos dados seriam tratados do seguinte modo:

- Caso a LIM esteja ativada, a informação seria repassada ao órgão governamental dedicado, o qual informaria ao proprietário pelo correio, de que seu veículo apresenta um problema que influencia as emissões de escapamento, e que é necessário que se tomem providências para a correção do problema em um prazo determinado sob pena de multa. Também seria informado à montadora, como dado estatístico ou para controle de garantia do produto, bem como à oficina de manutenção cadastrada pelo usuário.

- Caso o IUMPR de uma frota específica de veículos esteja abaixo do limite mínimo exigido pela norma, a informação seria enviada simultaneamente ao órgão governamental dedicado e à montadora para que se faça uma pesquisa específica nesta frota e se tome medidas corretivas necessárias.

A Figuras 12 e 13 ilustram como funcionaria a estrutura de transmissão dos dados de OBD dos veículos. 


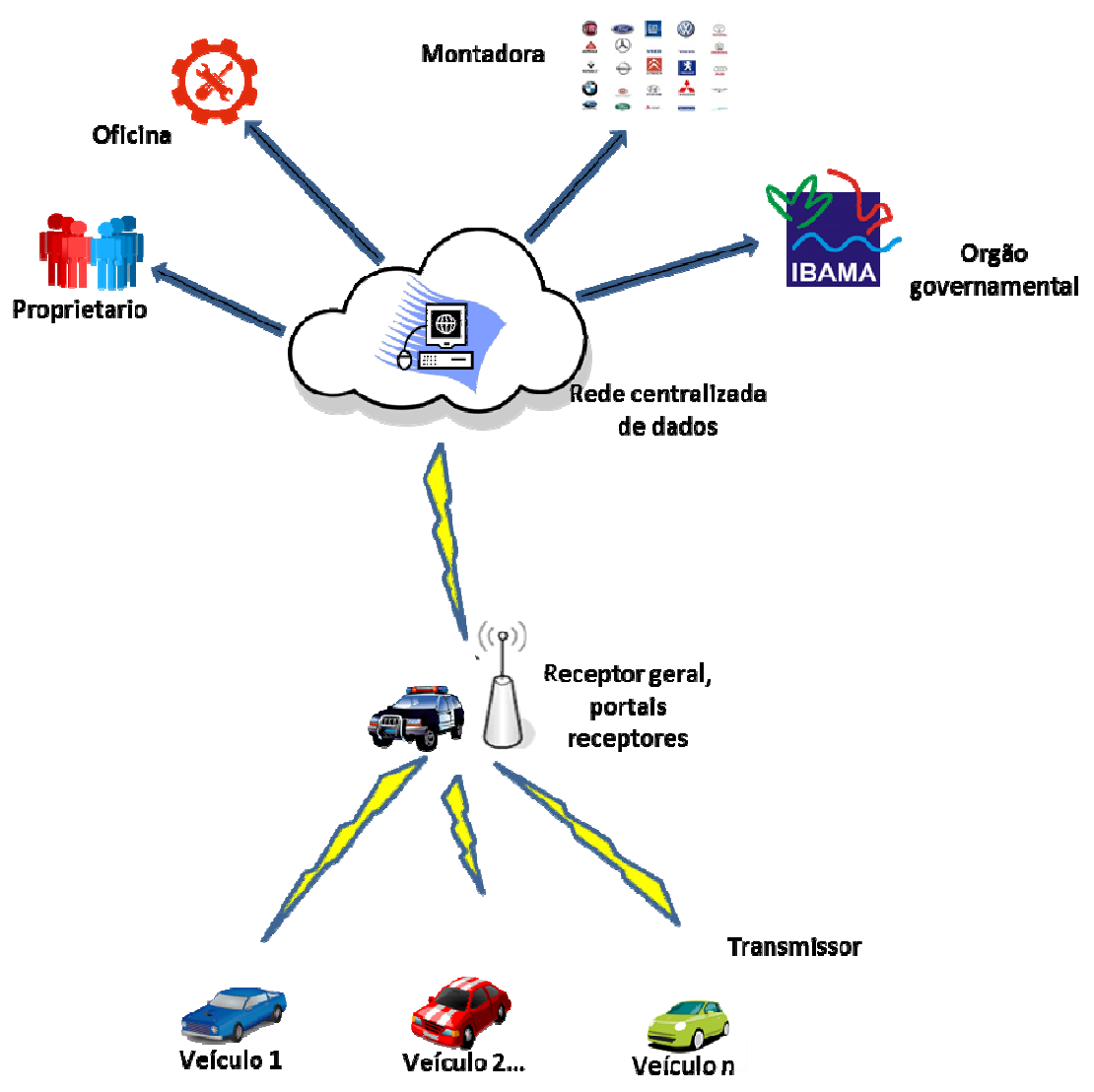

Figura 12. Esquema de comunicação para a transmissão de dados OBD

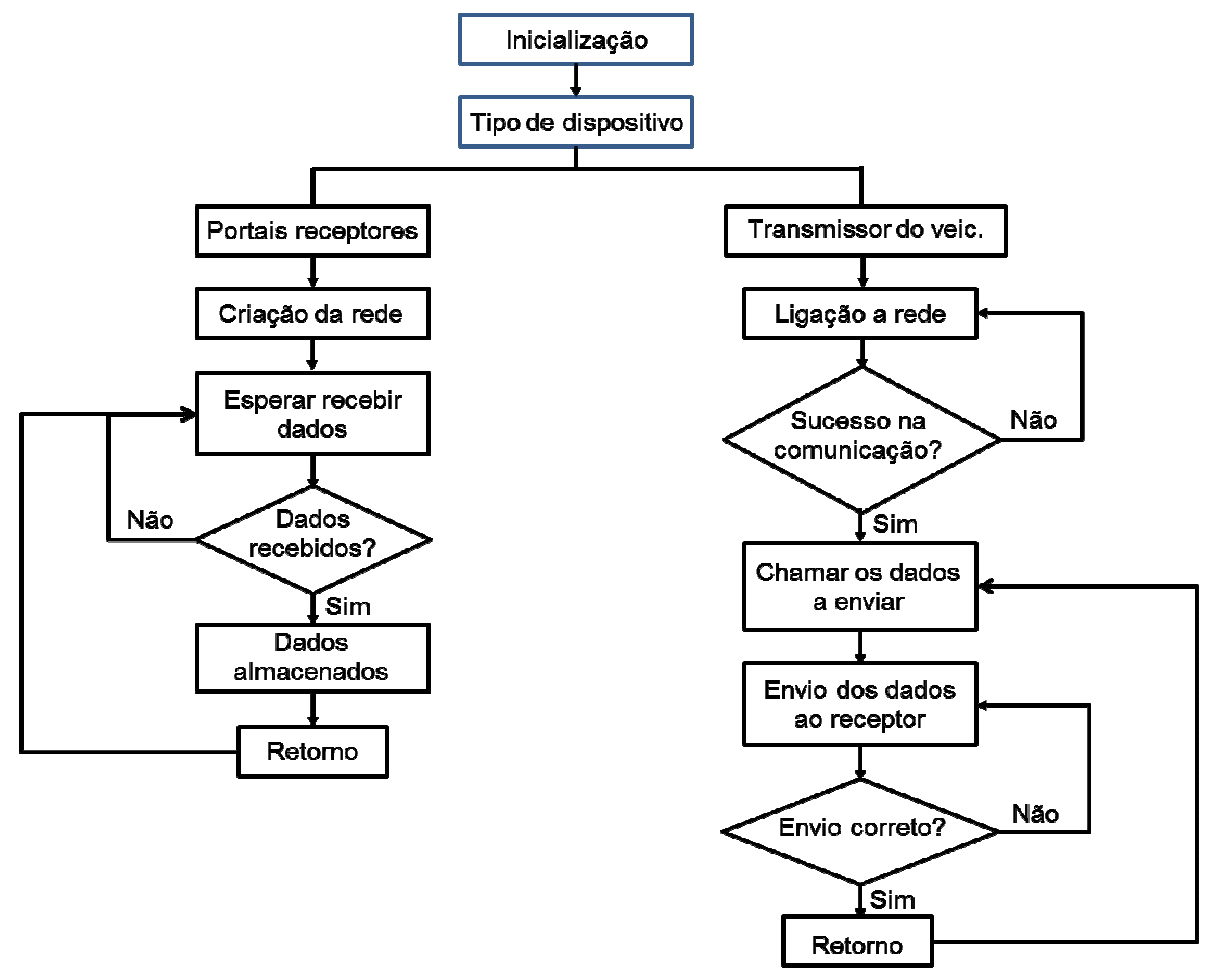

Figura 13. Diagrama de fluxo de dados na comunicação entre veículos e "portais receptores" 


\section{CONCLUSÕES}

O presente trabalho mostrou uma alternativa à modalidade tradicional de inspeção veicular, apresentando a transmissão dos resultados OBD (LIM e IUMPR), como ferramenta de avaliação dos sistemas de controle de emissões de poluentes. Isto beneficiaria as três partes interessadas:

- Usuário: Redução no custo e tempo dedicados às inspeções anuais convencionais;

- Órgãos governamentais: O contínuo monitoramento da frota evitaria que alguns veículos retornem às características originais de fábrica apenas para serem aprovados na inspeção e depois voltem a ser alterados; Por não se tratar de um sistema de elevada complexidade poderia ter abrangência nacional, o que traria a melhoria da qualidade do ar em todo o país;

- Montadora: Este sistema seria uma importante ferramenta de controle de qualidade de seus produtos, uma vez que poderia identificar oportunidades de melhoria em sistemas e componentes relacionados às emissões veiculares, ou mesmo identificar casos de mau uso do veículo, eximindo-a de qualquer responsabilidade durante a vigência da garantia do veículo

\section{ABREVIAÇÕES}

CARB

CONAMA

FTP

IBAMA

$\mathrm{I} / \mathrm{M}$

IUMPR

LEV

LIM

NEDC

OBD

PROCONVE

VIN
California Air Resources Board

Conselho Nacional de Meio Ambiente

Federal Test Procedure

Instituto Brasileiro do Meio Ambiente

Inspection and Maintenance

In Use Monitor Performance Ratio

Low Emission Vehicle

Lâmpada Indicadora de Mau Funcionamento

New European Drive Cycle

On Board Diagnostics

Programa de Controle da Poluição do Ar por Veículos Automotores

Vehicle Identification Number 


\section{REFERÊNCIAS}

[1] DUQUE, Oscar; ROEWER JR, Guido; FURLAN, Paolo; BUCCI, Alberto. “OBD no Brasil, suas particularidades e desafios futuros” XIX Simpósio Internacional de Engenharia Automotiva SIMEA 2011.

[2] MURGEL, Gabriel; CARDINALE, Fábio; SZWARC, Alfred. "Relatório de avaliação do programa IM-SP: Descritivas e resultados de veículos com motor do ciclo Otto em 2011”. São Paulo, junho de 2012.

[3] ZAMBON, Michel; BALTUSIS, Paul; BURGOS, Eduardo; CASSANIGA, Marinna. “Comparison and perspectives of Brazilian OBD and I/M". XVIII Simpósio Internacional de Engenharia Automotiva SIMEA 2010

[4] DANIEL, Ritchie; BROOKS, Thomas; PATES, David. "Analysis of EU and US Drive Styles to Improve Understanding of Market Usage and the Effects on OBD Monitor IUMPR”. Mahle Powertrain Ltd. SAE Paper No. 2009-01-0236

[5] DELPHI. "Worldwide Emission Standards, Passenger Cars and Light Duty Vehicles". 2012/2013

[6] VOLKSWAGEN A.G. “Selbststudienprogramm 230. Kfz-Abgasemissionen”. Wolfsburg. 2000

[7] “Av. da Emancipação - Hortolândia - São Paulo, 13184-654 para Americana - São Paulo”. Google Maps. Disponível em http://maps.google.com.br, Data de acesso: 23.04.2014

[8] FENG, Guosheng; FENG, Bo; JIA, Sumei; FENG, Lisha. "Development of an On-board Diagnostics System Based on Wireless Network". Sensors \& Transducers, Vol. 164, Issue 2, Feb. 2014. Disponível em http://www.sensorsportal.com/HTML/DIGEST/february_2014/Vol_164/P_1838.pdf. Data de acesso: 15.04 .2014 